\title{
Colon cancer metastasis to the mandibular gingiva with partial occult squamous differentiation: A case report and literature review
}

\author{
QUAN-GUANG REN ${ }^{1}$, TAO HUANG ${ }^{2}$, SHENG-LI YANG $^{1}$ and JIAN-LI HU ${ }^{1}$ \\ ${ }^{1}$ Cancer Center, Union Hospital, Tongji Medical College, Huazhong University of Science and Technology, \\ Wuhan, Hubei 430022; ${ }^{2}$ Department of Pediatrics, Tianyou Hospital, \\ Wuhan University of Science and Technology, Wuhan, Hubei 430064, P.R. China
}

Received September 7, 2016; Accepted November 18, 2016

DOI: $10.3892 / \operatorname{mco} .2016 .1102$

\begin{abstract}
Metastasis is the primary cause of death among patients with colon cancer. However, the number of available studies regarding oral cavity metastases from colon cancer is currently limited. We herein report an unusual case of a 60-year-old male patient who developed an oral cavity metastasis from colon cancer. A total of 12 clinical case studies reporting colon cancer metastases to the mandibular gingival region were also reviewed, with the aim to elucidate the clinical and pathological characteristics of this disease entity in order to improve clinical diagnosis and treatment. It was demonstrated that patients with oral cavity metastases from colon cancer were predominantly in the sixth or seventh decades of life. The mandible was the main site of metastatic tumors to the oral cavity, while the occurrence of gingival metastases was comparatively rare. Moreover, the diagnoses of an oral metastatic tumor and primary colon cancer were often synchronous and were frequently accompanied with metastases to other organs. Several key aspects were suggested that should be accounted for when diagnosing colon cancer patients, including focusing attention to oral symptoms when examining cancer patients, utilizing a multidisciplinary approach for differential diagnosis and utilizing postoperative pathological examination to accurately diagnose the type of tumor and optimize the efficacy of treatment.
\end{abstract}

Correspondence to: Dr Sheng-Li Yang or Professor Jian-Li Hu, Cancer Center, Union Hospital, Tongji Medical College, Huazhong University of Science and Technology, 1277 Jiefang Avenue, Wuhan, Hubei 430022, P.R. China

E-mail: yangshengli2014@yahoo.com

E-mail: j15199@126.com

Key words: colon cancer, oral cavity metastases, gingival region, adenocarcinoma, squamous differentiation

\section{Introduction}

Colon cancer is second in morbidity and the third cause of cancer-related mortality worldwide (1). With the advances in diagnostic techniques, early diagnosis of metastases in patients with colon cancer has also improved, allowing patients to access treatment timely and effectively (2). Hematogenous metastasis is the main route of metastasis of colon cancer cells (3). Hence, the metastatic cells are able to invade distal organs and structures. The most common site of metastatic tumors originating from colon cancer is the liver, followed by the lungs, bone and other internal organs (4). Although liver metastases from colon cancer has a relatively favorable prognosis (progression-free survival of 23 months), metastases from colon cancer remain to be further investigated $(4,5)$. Moreover, colon cancer metastases to rare sites, such as the oral cavity, carry a worse prognosis (6).

Metastatic oral tumors are rare, contributing to $\sim 1 \%$ of all oral tumors (7). Theoretically, all types of tumors may metastasize to the oral cavity, the most common primary organs being the lungs in men and the breast in women. However, metastatic tumors in the oral cavity originating from the colon are rare, and the number of relevant clinical studies is limited. The lack of clinical observations makes the diagnosis and treatment of oral metastases difficult. We herein report an unusual case of colon carcinoma, where the patient originally presented with oral bleeding, with ulceration and edema of the gingival region of the mandible. The patient was later diagnosed with a metastatic gingival tumor originating from a primary colon adenocarcinoma with partial squamous differentiation.

\section{Case report}

The patient, a 60-year-old male, is currently undergoing chemotherapy for colon adenocarcinoma, which was diagnosed in April, 2014. A radical operation was performed at the time for the adenocarcinoma; however, liver metastases from colon cancer appeared postoperatively (December, 2014). The patient subsequently received successive bouts of chemotherapy (FOLFOX4, XELIRI, FOLFIRI, Raltitrexed + irinotecan and S-1 + apatinib) and radiotherapy. 

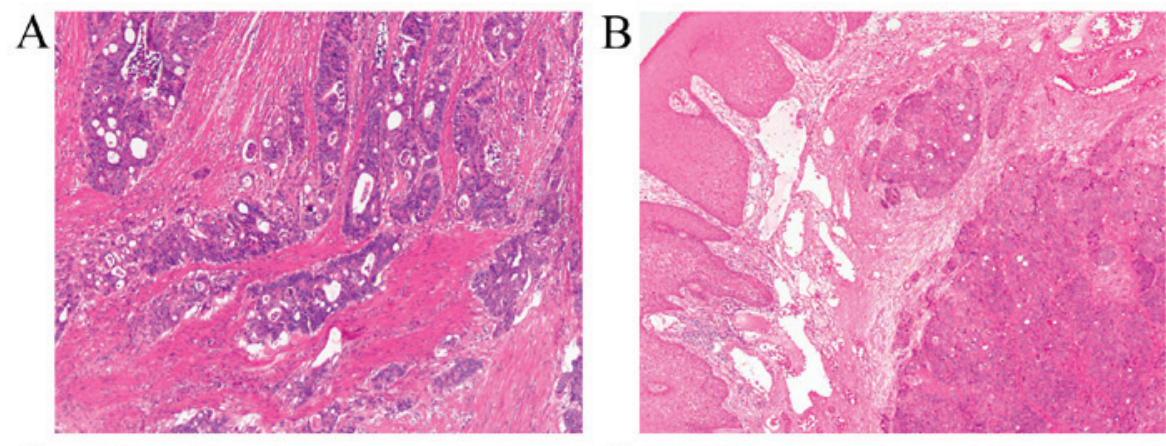

$\mathrm{C}$
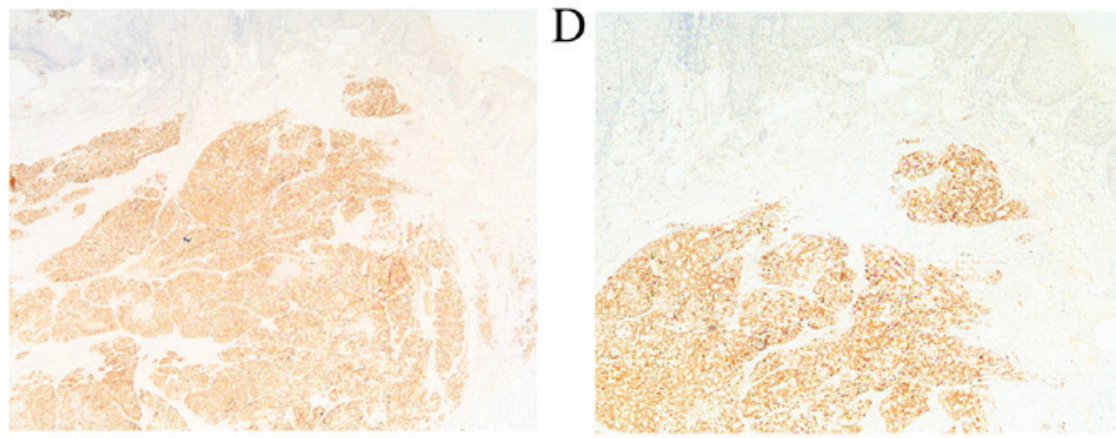

Figure 1. Representative images of the primary carcinoma and metastatic lesion. (A) Histologically, the colon neoplasm was a poorly differentiated adenocarcinoma (hematoxylin and eosin staining; original magnification, x40). (B) Histologically, the gingival neoplasm was a poorly differentiated adenocarcinoma (hematoxylin and eosin staining; original magnification, $\mathrm{x} 40$ ). (C and D) On immunohistochemical examination, the cancer cells of the gingival lesion were positive for CDX2 [original magnification, (C) x20 and (D) x40)].

The metastatic lesions were treated with injections of anhydrous alcohol and ultrasound-guided radiofrequency ablation, but the cancer progressed (increase in the size of the hepatic lesions and the levels of carcinoembryonic antigen and carbohydrate antigen 19-9). In May 2016, the patient complained of oral bleeding, ulceration and edema of the left gingival region of the mandible over several weeks. The patient was originally prescribed oral metronidazole by an oral clinician. As the aforementioned symptoms were not relieved with this treatment, the patient reported the symptoms to his doctor at the Cancer Center of the Union Hospital (Wuhan, China) in June, 2016, during his chemotherapy session for colon cancer. An initial extraoral examination yielded no significant findings, and the patient had no family history of oral diseases. However, a reddish nodule was found in the gingival mucosa of the left mandible, and the patient also reported pain and looseness of the teeth in the vicinity of the nodule. The patient was referred to our oral clinician for a consultation. It was suggested that proliferative response to inflammation, benign neoplasms and primary or metastatic tumors of the oral cavity should be considered in the differential diagnosis. During that time, the patient continued to receive the scheduled chemotherapy. With the suspicion of oral cavity metastases from the colon adenocarcinoma, an excisional biopsy was performed. The result of the pathological examination revealed poorly differentiated adenocarcinoma, indicative of metastatic adenocarcinoma originating in the colon, with partial squamous cell differentiation (Fig. 1). The patient was ultimately diagnosed with gingival metastases from the colon adenocarcinoma with partial squamous differentiation. Chemotherapy and local radiotherapy of the left gingival region was then scheduled.

\section{Discussion}

Despite advances in medicine, the incidence and mortality of colon cancer are on the increase in developing countries. Moreover, once metastases occur, tolerance to treatment commonly develops, which is associated with a poor prognosis $(1,8)$. As metastasis of colon cancer to the oral cavity is rare, clinical observations and studies are scarce. To the best of our knowledge, this is the first case of oral cavity metastases from colon adenocarcinoma to be accompanied by partial squamous differentiation.

On immunohistochemical examination, the tumor cells of the gingival lesion were positive for cytokeratin (CK)20, villin, CDX2, P63, P40, CK5/6, and sporadically for CK7, whereas they were negative for synaptophysin and chromogranin A. Tumor cells expressing CDX2 and villin were hypothesized to have originated from the digestive tract, while positive immunostaining for CK20 and sporadic CK7 positivity aided in the preliminarily confirmation of metastases from colon cancer (9). Of note, CK5/6, P40 and P63, which are biomarkers for squamous carcinomas, were also highly expressed. Finally, the definitive diagnosis was gingival metastasis from sigmoid adenocarcinoma with partial occult squamous differentiation. To the best of our knowledge, this is the first reported case of this type of gingival metastasis. While the origin of the gingival metastases from the colon adenocarcinoma was confirmed, the presence of the partial squamous differentiation in the gingival lesion has not been fully elucidated. This finding may be attributed to squamous differentiation of the adenocarcinoma; however, there is also a possibility of a second malignancy, particularly since the majority of primary cancers of the head and neck (including 


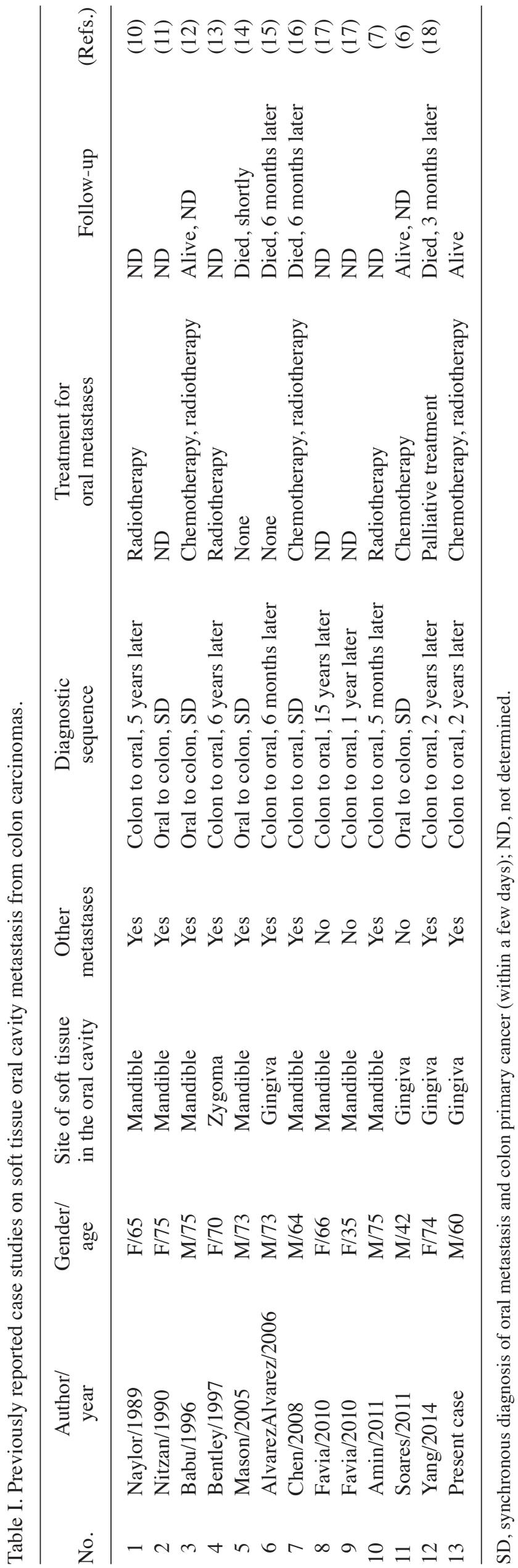

the oral cavity) are squamous cell carcinomas. At present, in addition to continuing chemotherapy, the patient was scheduled for local radiotherapy to the gingival region.

To better understand the association between oral cavity metastases and colon cancer, 12 cases of oral cavity metastases of colon cancer, including the present case, were reviewed [6,7,10-18); Table I]. It was observed that mandibular metastases from colon cancer regularly occurred the sixth and seventh decades of life. Oral metastases in patients with colon cancer may be correlated with age, whereas there was no significant difference according to gender. There is a well-known age-associated decline in function of the immune system; in addition, the immune state of the patients and inflammation have been suggested to play an important role in the invasion and metastasis of tumor cells $(19,20)$. Therefore, it is hypothesized that a reduced immune response may contribute to the higher incidence of oral cavity metastases from colon cancer. It was also observed that the most common site of oral metastases in colon cancer patients was the mandible (8/13), followed by the gingiva, accounting for one-fourth of all oral metastases (4/13). Approximately one-fourth (5/13) of the reviewed cases reported synchronous diagnosis of oral cavity metastases and primary colon cancer. Of note, colon cancer also metastasized to extraoral tissues and organs, in addition to the oral cavity. Furthermore, it appears that the liver is the first organ to develop metastasis from colon cancer, followed by the lungs, bone and other rare sites. A potential underlying mechanism may include specific tissues and organs releasing respective ligands to attract metastatic cancer cells of differential origins. Furthermore, patients with oral metastases responded poorly to treatment and were unable tolerate follow-up treatment.

We herein report a rare case of oral metastasis from a colon adenocarcinoma with partial squamous differentiation. Based on our observations and the review of similar case studies to highlight the general characteristics of oral metastases in cancer patients, several key points should be accounted for when diagnosing colon cancer patients. First, attention should be focused on oral symptoms, such as gingival bleeding, ulcerations and abscesses, when examining cancer patients, particularly elderly patients. Clinical examination and past medical history should also be carefully evaluated, utilizing a multidisciplinary approach to differential diagnosis to ensure the most comprehensive assessment of the patients. Most importantly, excisional biopsies and pathological examination are invaluable for accurately diagnosing the type of tumor, in order to optimize the efficacy of treatment for adenocarcinomas and squamous carcinomas and improve survival time and quality of life prior to the onset of cachexia. Incorporating these aspects in diagnosis will assist clinical physicians in making accurate diagnoses, ensuring a better prognosis.

\section{References}

1. Torre LA, Bray F, Siegel RL, Ferlay J, Lortet-Tieulent J and Jemal A: Global cancer statistics, 2012. CA Cancer J Clin 65: $87-108,2015$

2. Miller KD, Siegel RL, Lin CC, Mariotto AB, Kramer JL, Rowland JH, Stein KD, Alteri R and Jemal A: Cancer treatment and survivorship statistics, 2016. CA Cancer J Clin 66: 271-289, 2016. 
3. Gutman M and Fidler IJ: Biology of human colon cancer metastasis. World J Surg 19: 226-234, 1995.

4. Riihimäki M, Hemminki A, Sundquist J and Hemminki K: Patterns of metastasis in colon and rectal cancer. Sci Rep 6 : 29765, 2016.

5. Bruera G, Cannita K, Giuliante F, Lanfiuti Baldi P, Vicentini R, Marchetti P, Nuzzo G, Antonucci A, Ficorella C and Ricevuto E: Effectiveness of liver metastasectomies in patients with metastatic colorectal cancer treated with FIr-B/FOx triplet chemotherapy plus bevacizumab. Clin Colorectal Cancer 11: 119-126, 2012.

6. Soares AB, Thomaz LA, Duarte MT, de Camargo de Moraes P and de Araújo VC: Metastatic adenocarcinoma of the colon: Early manifestation in gingival tissue. Head Neck Pathol 5: 140-143, 2011.

7. Amin A, Jha M and Reddy A: Lower lip numbness in a patient with colorectal cancer. BMJ case Rep 2011: pii: bcr0120113682, 2011.

8. Ukegjini K, Zadnikar M, Warschkow R, Müller S, Schmied BM and Marti L: Baseline mortality-adjusted survival in colon cancer patients. Langenbecks Arch Surg 401: 633-641, 2016.

9. Meer S and Altini M: CK7+/CK20-immunoexpression profile is typical of salivary gland neoplasia. Histopathology 51: 26-32, 2007.

10. Naylor GD, Auclair PL, Rathbun WA and Hall EH: Metastatic adenocarcinoma of the colon presenting as periradicular periodontal disease: A case report. Oral Surg Oral Med Oral Pathol 67: 162-166, 1989.

11. Nitzan DW, Livni N, Marmary Y, Ben-Baruch N, Sela J and Catane R: The use of monoclonal anti-CEA antibody immunohistochemistry in detecting the origin of oral cavity metastasis. Int J Oral Maxillofac Surg 19: 162-164, 1990.
12. Babu KG, Raud C, Kumaraswamy SV and Lalitha N: Carcinoma colon with mandible and liver metastases. Br J Oral Maxillofac Surg 34: 133-134, 1996

13. Bentley RP and Worrall SF: Carcinoma of the colon with mandible and liver metastases. Br J Oral Maxillofac Surg 35: 221-222, 1997.

14. Mason AC, Azari KK, Farkas LM, Duvvuri U and Myers EN: Metastatic adenocarcinoma of the colon presenting as a mass in the mandible. Head Neck 27: 729-732, 2005.

15. Alvarez-Alvarez C, Iglesias-Rodríguez B, Pazo-Irazu S and Delgado-Sánchez-Gracián C: Colonic adenocarcinoma with metastasis to the gingiva. Med Oral Patol Oral Cir Bucal 11: E85-E87, 2006 (In English, Spanish).

16. Chen HL, Chang WH, Shih SC, Pang KK and Bair MJ: Trismus and trigeminal neuralgia in one patient with colon cancer. J Natl Med Assoc 100: 740-742, 2008.

17. Favia G, Maiorano E and Lo Muzio L: Gingival metastasis from colonic adenocarcinoma. Clin Gastroenterol Hepatol 8: A28, 2010.

18. Yang RH, Chu YK and Li WY: Unusual site of metastasis detected with FDG PET/CT in a case of recurrent rectosigmoid cancer. Clin Nucl Med 39: 355-357, 2014.

19. Mager LF, Wasmer MH, Rau TT and Krebs P: Cytokine-induced modulation of colorectal cancer. Front Oncol 6: 96, 2016.

20. Koh SJ, Kim JM, Kim IK, Ko SH and Kim JS: Anti-inflammatory mechanism of metformin and its effects in intestinal inflammation and colitis-associated colon cancer. J Gastroenterol Hepatol 29: 502-510, 2014. 